\title{
The Effect of EFT (Emotional Freedom Techniques) on Athletic Performance: A Randomized Controlled Blind Trial
}

\author{
Dawson Church*
}

Foundation for Epigenetic Medicine, 1490 Mark West Springs Rd, Santa Rosa CA 95404, USA

\begin{abstract}
The present study investigates the effect of a psychophysiological intervention, Emotional Freedom Techniques (EFT), on athletic performance. It evaluates whether a single EFT treatment can produce an improvement in highperformance men's and women's PAC-10 college basketball team members $(n=26)$. The treatment group received a 15 minute EFT session while a performance-matched attention control group received a placebo intervention of similar duration. Performance was measured on free throws and vertical jump height. The time frame of data collection and treatment simulated an actual basketball game. A statistically significant difference between the two groups was found for free throws $(\mathrm{p}<.03)$. On post-test, players who received the EFT intervention improved an average of $20.8 \%$, while the attention control group decreased an average of $16.6 \%$. There was no difference between treatment groups in jump height. When performance was analyzed separately by gender, trends toward significance were found for the women's team on both performance measures with better results for the EFT intervention group. This indicates that EFT performed as an intervention during the course of an athletic event may improve free throw performance.
\end{abstract}

Keywords: Basketball, performance, free throws, Emotional Freedom Techniques (EFT), anxiety, stress.

\section{INTRODUCTION}

There have been numerous studies of the effect of Emotional Freedom Techniques (EFT) and other Energy Psychology (EP) therapies for various psychological conditions, including depression, anxiety, phobias, and posttraumatic stress disorder [1]. However, no prior study has examined the effect of EP on healthy and high-performing individuals, though news media have reported increasing use of EFT among elite athletes [2-4]. It is practiced as a very brief intervention before or during the course of a game, and athletes typically report immediately improved performance.

EFT was developed by Gary Craig as an abbreviation of the methods used in Thought Field Therapy (TFT), an earlier Energy Psychology method that used to elaborate diagnostic and treatment protocols [5]. Craig came to the conclusion that a brief and standardized protocol could treat many psychological conditions without the need for lengthy diagnoses, and organized these points into a easily-administered method [6]. EFT is uniformly applied by practitioners; The EFT Manual [7] has been available as a free web download since the 1990s, and a consensus EFT research protocol has been used for prior studies. In essence, EFT consists of a subject recalling an emotionally traumatic event, measuring its intensity, pairing the recall with a self-acceptance statement, stimulating 13 specific points on the body which are claimed to produce a calming response, and then retesting intensity to determine if the intervention is reducing stress. The entire process takes between 30 and 60 seconds.

*Address correspondence to this author at the Foundation for Epigenetic Medicine, 1490 Mark West Springs Rd, Santa Rosa CA 95404, USA; Tel: 707-237-6951; E-mail: dawson@soulmedicine.net
A number of championship athletes in a variety of sports report immediate and large gains after EFT [8, 9]. Published studies have found the technique effective for several types of psychological distress, such as anxiety [10], phobias [11, 12], and PTSD [13]. Most of these studies use a single session of EFT, and the effects of EFT have been found to hold during follow up periods ranging from one month to one year $[10,12]$. Therapists using Energy Psychology report an absence of adverse events and the retraumatization that can be associated with the recall of traumatic events [13-15]. Numerous reports on the EFT web site document its use by coaches in the field as well as licensed professionals in the office, and indicate that self-application can be efficacious. Clinical reports also suggest that by reducing emotional trauma, EFT can affect physical conditions including joint pains, allergies, chronic fatigue syndrome, rashes, arthritis, colds, flu, and toothaches [6]. A randomized blind clinical trial has found EFT to be effective for fibromyalgia pain and muscular aches [16].

There is considerable debate as to the mechanisms of action of EFT and other Energy Psychology therapies. Craig and other proponents of EFT believe that the method is effective because its 13 points are on "energy meridians" corresponding to the endpoints of the acupuncture meridians described by traditional Chinese medicine [17]. However, other authors prefer conventional physiological mechanisms to explain the efficacy of Energy Psychology. These include the deactivation of the hyperarousal of the amygdala and other fight-or-flight structures in the brain that occurs during traumatic recall [1]; the semiconductive properties noted in connective tissue when a piezoelectric stimulus is produced by manual stimulation such as that used in EFT [18]; stimulation of the rapid reuptake and resynthesis of stress hor- 
mones such as cortisol [19]; upregulation of the secretion of inhibitory neurotransmitters such as serotonin, along with downregulation of excitatory ones such as dopamine [6]; termination of the alarm response in the Sympathetic Nervous System, and replacement with a relaxation response in the Parasympathetic Nervous System [20]; the release of serotonin in the prefrontal cortex of the brain [21], and enhanced expression of stress-dampening Immediate Early Genes (IEGs) in brain structures such as the hippocampus and hypothalamus, with corresponding regulation of the HPA axis [22]. These mechanisms of action, working together or separately, have been posited as explanations for the extremely fast responses obtained in Energy Psychology treatments.

In the present study, EFT was administered under randomized controlled blind conditions to determine its effect on two specific skills relating to the game of basketball: percent of free throws, and jump height. The primary hypothesis being tested was that EFT would mitigate the effects of stress, thus improving athletic performance. Treatment was the independent variable, with jump height and free throw accuracy as the dependent variables. Other variables such as the passage of time, fatigue, gender, and baseline skill were controlled for by the design of the study. The purpose of this study was to evaluate whether EFT could make a difference in elite athletes by treating any stress or anxiety associated with sports performance, using a replicable design of sound methodological quality.

Analysis has shown that accurately performed free throws are one of the most critical factors in winning basketball games [23], leading to performance stress on players being asked to perform this maneuver. Free throws have been used in more than two dozen studies to measure the effects of treatments such as Cognitive Behavioral Therapy (CBT) on reducing anxiety and increasing athletic performance [24-26]. Components of EFT resemble CBT in that they seek to shift anxiety-producing cognitions in subjects. A test of anxiety in competitive tennis players found that winners scored significantly lower for both cognitive anxiety and somatic anxiety; their concentrations of salivary cortisol, a physiological marker for stress, were also lower [27].

The opportunity to study basketball athletes resulted from the adoption of EFT by the baseball team of the university, some members of whom had learned EFT two years prior, after which the team won two consecutive national college championships [28]. The game of basketball was chosen because psychological stress has been demonstrated to affect free throw performance [29], and a repeated-measures experimental design permitted empirical determination of performance changes in a compressed time frame.

\section{METHOD}

\section{Participants and Procedures}

Participants in the present study were members of the men's and women's basketball teams at an elite PAC-10 institution. After approval by the university, the study was performed on-campus, and data gathering took place during the course a half-day session with the men and a second halfday with the women. Informed consent was obtained from participants. A total of 26 players participated, 14 male and 12 female.

All participants performed their regular warm-up period, to eliminate any effect on athletic performance of inadequate muscular preparation. The warm-up was of a nature and duration standard to the teams, lasting approximately ten minutes and consisting of stretches and running.

All subjects then performed ten free throws, and two groups of three jumps (six jumps total). They were then randomized by an independent research assistant into two matched groups, based on the average height of the second group of three jumps. In order to control for investigator expectancy, randomization and matching of the teams, and data recording, was done by research assistants without applicable experience of either condition.

The vertical height of the subjects' jumps was measured using a Probotics jump pad (www.Probotics.org). This device electronically records and displays jump height in tenths of an inch (for example, 265 units $=26.5$ inches). The free throw test consisted of ten throws to the basket from the free throw line on the court. Other members of the team observed the participants while the jumps and free throws were being recorded. Prior research using free throws as a measure has suggested that human performance changes when under the stress of observation by others $[29,30]$.

Following randomization, the two teams were sent to opposite ends of the basketball court. In order to preserve blindness, they were not permitted to talk to members of the other team. They were instructed to practice free throws, run, dribble, do vertical jumps, and generally stay warmed up for the duration of the study. This was done to counteract any confounding effect due to body cooling between measurements, and to simulate the fatigue over time that players might experience during the course of an actual basketball game. The effects of fatigue on both the muscular and nervous system, and the subsequent degradation of performance during the course of a game, have been described by other researchers $[31,32]$. Athletes were also instructed to drink water, since dehydration has been shown in other studies to affect free throw performance [33, 34].

The experimental treatment consisted of fifteen minutes of EFT. As an attention control, a blind placebo comparison group received an inspirational reading of tips and techniques from a former college basketball coach, Rick Pitino [35]. It covered topics such as setting high personal standards, improving with practice, building self-confidence, positive thinking, handling stress well, and the value of hard work. These topics provided the control group with a brief, multi-dimensional positive cognitive stimulus, but without the somatic element of tapping present in EFT. Both experimental and control interventions were performed in a tenminute first session and a five-minute follow-up session. The time lapse between the first test of the first participant and the last test of the last participant was approximately two hours. Compressing the data gathering and treatment into two hours was designed to approximate the time period of an actual basketball game.

For treatment, each of the teams were taken as a group to a different location in the building. They were then told the nature of the intervention they were to receive. The first par- 
ticipants were randomly selected from each team and the rest of the team members were asked to return to their end of the basketball court and to "stay warm." This was accomplished by the performance of drills and impromptu games, in order to produce the same degree of progressive muscle fatigue and performance degradation noted during regular games $[36,37]$.

In both the experimental and control groups, treatments were then administered individually. A participant was taken into a private office, and the first ten minute treatment was administered. Participants then went back to the court, performed three vertical jumps, and returned to the treatment room for an additional treatment lasting five minutes. After this, they returned to the court to perform three final jumps and a post-treatment set of ten free throws. This process continued until all team members were treated.

\section{Treatment Intervention}

EFT is a brief exposure therapy that combines both cognitive and somatic elements. In the first step of the EFT session, participants recall an emotionally disturbing incident, e.g. "When I was in first grade, my dad got mad and spanked me when I said I didn't want to play T-ball." They selfassess the degree of emotional distress associated with this incident on an 11 point Subjective Units of Distress (SUD) scale [38]. On the SUD scale, zero represents no problem, and ten represents the most pressing experience of the problem. In the case of physical pain, for instance, zero represents no pain, while ten represents the most extreme pain imaginable. In the case of emotional pain, the subject is asked to identify a point in the body particularly identified with the pain, and to note its SUD score.

After deriving a SUD score, participants create a short verbal summary of the problem they wish to change, and combine it with a statement of self-acceptance. In EFT this is referred to as the "setup statement." A setup statement pairs the problem with acceptance, e.g., "Even though I have (this problem), I fully and completely accept myself." Examples of setup statements are, "Even though I'm angry when I think about dad beating me, I fully and completely accept myself," or, "Even though I'm worried the other guys will gang up on me if I throw ten in a row in the basket..." or "Even though I worry that I'll tear a hamstring if I jump too high..."

Following this cognitive element, the somatic portion is administered, either by the individual or a therapist. This involves tapping with the fingertips of one hand on thirteen parts of the body, referred to as a "round" of "tapping." These correspond to the endpoints of acupuncture meridians in Traditional Chinese Medicine (TCM), and are used in acupuncture needling to reduce stress and treat various physical imbalances. EFT is described by its founder as "acupuncture without needles" [7]. Applying pressure to acupuncture points, instead of needling them, is used in traditional therapies such as Shiatsu massage, and has been found to have efficacy similar to needling [39].

The first point is the fleshy outside edge of the hand, called, for convenience, the "karate chop point." The participant taps this portion of the opposing hand repeatedly, while repeating the setup statement three times. Next five points on the face are tapped about seven times: the inner terminus of either eyebrow, the side of the eye socket, the center of the bony ridge below the eye, the middle of the upper lip just below the nose, and the center of the chin.

Then two points on the torso are tapped: the junction of collarbone and sternum, and the ribcage under the arms. Lastly, five points on the hand are tapped, the part of the cuticle of each finger closest the body, with the exception of the ring finger, and finally the groove between the bones of the last two fingers on the back of the hand.

After a round of tapping, subjects provide a second SUD score. Subjects typically report a decrease in the distress associated with a negative emotion or traumatic event after rubbing or tapping these points, e.g. "My anger was an eight but now it's a three." Two or more applications of EFT may be used to further reduce the SUD score. In this study, most participants completed one or two rounds of tapping in the first (ten minute) session, and a single round in the second (five minute) session.

In interviews with the athletes during this study, it was determined that their stress centered on issues involving their athletic and life problems, and that these factors were selfperceived to be affecting their game. There were four common treatment problems, and "setup statements" tended to focus on (a) limiting beliefs about performance, (b) anxieties in general, (c) negative past experiences, or (d) muscle memory of past maximum achievement.

Typical problems were tiredness, stress from facing exams, homework or poor grades, and family conflicts. Some issues were general and global beliefs: "I'm a poor athlete, but the team needs me because I shoot well." Others were very specific, "My upper body is strong, but my legs are weak. I've never been a good jumper." Some were from long ago, "My father went to jail when I was five, and I never saw him again," and others were from the present, "I'm worried about being here because I have a huge amount of homework to finish today." Thus a setup statement for a negative belief might be: "Even though I have weak legs, I fully and completely accept myself." A setup statement for a desired condition might be, "My legs are powerful and strong."

The goal of EFT treatment was to reduce the SUD level for one or more such issues during the short course of treatment, reduce the impact of the identified traumatic event, administer the somatic portion of treatment, and measure the results. Alternatively, EFT was performed while imagining a positive desired outcome, such as "I know deep inside I have the ability to dunk the ball every time." Experimental and control interventions were performed by Licensed Professional Counselors (LPCs). Data analysis was subsequently performed blind, by an independent research psychologist.

\section{RESULTS}

\section{Statistical Analysis}

Percent change scores for number of free throws and jump height were calculated to determine the amount of improvement or decline from pre-test. Independent samples two-tailed t-tests were conducted on the percent change score to determine if there were significant group differences. The t-tests analyses were also repeated separately for 
males and females. Baseline descriptive statistics for free throws and jump height by treatment condition and gender are presented in Table $\mathbf{1}$.

\section{Free Throw Results}

A statistically significant difference between the treatment groups was found $(\mathrm{t}(24)=-2.34, \mathrm{p}<.03)$. The athletes treated with EFT improved their free throw performance by an average of $20.8 \%$, while the control group decreased by an average of $16.6 \%$.

When the analyses were conducted within gender, there was a trend towards significance for females $(\mathrm{t}(10)=-1.96$, $\mathrm{p}<.08$ ). Free throws increased an average of $2.6 \%$ for females in the EFT treatment group while the attention control group decreased an average of $22.2 \%$. There was no treatment group difference for males. Results are presented in Table 2.

\section{Jump Height Results}

There was no statistically significant difference between the treatment and attention control groups on the percent change in jump height for the whole sample $(\mathrm{t}(12)=-0.79$, $\mathrm{p}<.47)$ and for males when analyzed separately $(\mathrm{t}(12)=$ $0.429, \mathrm{p}<.68)$. However there was a trend towards significance for the females $(\mathrm{t}(5.9)=-2.13, \mathrm{p}<.08)$. Females in the treatment group showed close to no change in jump height (decreased $0.73 \%$,), while females in the control group decreased 4.3\%. Results are presented in Table 2 .

\section{DISCUSSION}

Most studies of EFT and other forms of Energy Psychology have focused on psychological distress. Conditions such as phobias and anxiety have been the object of considerable examination and evaluation, and these therapies have been studied in even extremes of distress such as holocaust victims in Rwanda and ethnic cleansing victims in Kosovo [40]. However, no studies have yet been conducted of the effects of EFT on athletic performance, even though the brief nature of this intervention (typically under a minute) makes it more suited to application during stressful situations than therapies that require a series of office visits. Such studies are timely, given the many reports from coaches and athletes of EFT being applied in professional and college level golf, baseball, and American football [2].

Table 1. Free Throw and Jump Height Pre- and Posttest Means and Standard Deviations

\begin{tabular}{|c|c|c|c|c|c|c|}
\hline \multirow[b]{2}{*}{ Variable/Sample } & \multirow[b]{2}{*}{$\mathbf{N}$} & \multicolumn{2}{|c|}{ EFT Group } & \multirow[b]{2}{*}{$\mathbf{N}$} & \multicolumn{2}{|c|}{ Attention Control } \\
\hline & & Pretest Mean \pm SD & Posttest Mean \pm SD & & Pretest Mean \pm SD & Posttest Mean \pm SD \\
\hline Females & 6 & $8.00 \pm 2.00$ & $7.83 \pm 1.33$ & 6 & $8.50 \pm 1.38$ & $6.50 \pm 1.23$ \\
\hline Males & 7 & $6.71 \pm 2.81$ & $7.71 \pm 1.38$ & 7 & $7.57 \pm 1.62$ & $6.71 \pm 2.63$ \\
\hline \multicolumn{7}{|l|}{ Jump Height } \\
\hline Females & 6 & $183.00 \pm 24.75$ & $181.83 \pm 26.20$ & 6 & $189.78 \pm 30.14$ & $181.17 \pm 26.41$ \\
\hline Males & 7 & $278.29 \pm 27.36$ & $274.21 \pm 33.88$ & 7 & $270.38 \pm 36.10$ & $268.40 \pm 35.06$ \\
\hline Total sample & 13 & $234.31 \pm 55.44$ & $233.58 \pm 56.19$ & 13 & $233.18 \pm 52.72$ & $228.14 \pm 54.35$ \\
\hline
\end{tabular}

Table 2. Free Throw and Jump Height Percent Change T-test Means and Standard Deviations

\begin{tabular}{|l|l|l|l|l|l|l|l|}
\hline & \multicolumn{2}{|c|}{ EFT Group } & \multicolumn{3}{c|}{ Attention Control } \\
\hline Variable/Sample & N & Percent Change Mean \pm SD & N & Percent Change Mean \pm SD & Statistic & Significance \\
\hline \hline Free Throws & 6 & $2.59 \pm 25.90$ & 6 & $-22.17 \pm 16.94$ & $\mathrm{t}(10)=-1.96$ & $\mathrm{p}<.08$ \\
\hline Females & 7 & $36.47 \pm 66.62$ & 7 & $-11.73 \pm 26.82$ & $\mathrm{t}(7.9)=-1.78$ & $\mathrm{p}<.11$ \\
\hline Males & 13 & $20.83 \pm 52.98$ & 13 & $-16.55 \pm 22.55$ & $\mathrm{t}(24)=-2.34$ & $\mathrm{p}<.03$ \\
\hline Total sample & 6 & $-0.73 \pm 1.20$ & 6 & $-4.33 \pm 3.97$ & $\mathrm{t}(5.9)=-2.13$ & $\mathrm{p}<.08$ \\
\hline Jump Height & 6 & $-1.62 \pm 4.17$ & 7 & $-0.61 \pm 4.65$ & $\mathrm{t}(12)=0.43$ & $\mathrm{p}<.68$ \\
\hline Females & 7 & $-1.21 \pm 3.08$ & 13 & $-2.33 \pm 4.60$ & $\mathrm{t}(24)=-0.73$ & $\mathrm{p}<.47$ \\
\hline Males & 13 & & & \\
\hline Total sample & & & & \\
\hline
\end{tabular}


A limitation of this study is its small sample size, $n=26$. To compensate, statistical measures intended to compensate for individual variance were employed. When analyzing small samples, t-tests are used to account for degrees of freedom in the sample size. The above results fall within the parameters of the statistical tests chosen to analyze them. However, replication in larger subject populations is required to see if these results are typical.

The results raise some additional questions. Why did vertical jump height not improve to a statistically significant degree, while free throws did? Jump height may be less dependent on emotional states, and more dependent on physiological factors, such as physical conditioning, flexibility, and strength; while free throws may be more affected by emotional factors, such as stress. There is evidence to suggest that players with the ability to perform consistently under stress have better free throw percentages [29].

Jump height performance improvements might require longer EFT sessions. Established therapies like Cognitive Behavioral Therapy or CBT usually prescribe between 6 and 12 one-hour sessions. A study using CBT for jump height improvement found that as players moved from negative cognitions such as the limiting setup statements above, to positive ones, their jump height improved significantly [25]. Another study of CBT also found improvement in free throw percentages after treatment, though again, between 7 and 9 hours of treatment were required [26]. The quality of selftalk (mediated by the self-acceptance statement of EFTs setup phrase) has also been found to affect free throw scores [41]. All these studies used courses of therapy much longer than a 15 minute format. The clinicians administering EFT during this study speculated that to have an effect on jump height, they would have required 60 minute therapeutic sessions, or even several sessions. They noted the same effects as Mollon [14] and Schultz [15] that rather than recall of traumatic memories resulting in symptoms of retraumatization, the degree of emotional distress in subjects decreased as they tapped. A replication might test the hypothesis that longer sessions correlate with a decrease in symptomatology. A replication should also match teams based on free throws, not jump height, since that is where statistical significance was strongest in this study. A replication might also use a double blind design, with both therapists and subjects being blind, though the practical challenges of maintaining therapist blindness (and hence controlling for therapist expectancy during treatment, as well as during data collection as was done during the present study) are considerable. With a design otherwise similar to this study, a therapist blind would in effect produce the robust structure of a triple-blind study, since, in the present case, data collection and analysis was done blind. A replication might also use a brief anxiety instrument, such as the Beck Anxiety Inventory, to numerically assess any correlation between anxiety score drops and performance increases.

The discharge of emotional trauma has been linked to athletic performance by other scholars $[42,43]$. The contrary hypothesis has been proposed: that athletes who are angry and anxious may be able to funnel that energy to produce greater vigor during play. However, the study of basketball teams has shown that emotional equilibrium and calm judgment contribute to factors that determine success in basket- ball, such as accuracy and sensitive teamwork [23]. Consistently good players are able to perform well even under stress. The ability to discharge stress and stay focused allows an athlete's full skill set to come to bear on the athletic task at hand, and EFT is used by some athletes for this purpose [8].

Studies of non-athlete populations note a decrease in stress after EFT. Decreased levels of anxiety, depression and other conditions occur, with gains maintained on follow up [10]. Outcome studies of EFT in clinical settings have shown a large effect size, and efficacy in a wide variety of psychological conditions [1]. Studies of physiological markers of stress, such as levels of the stress hormone cortisol [19], as well as pain and joint range of motion [44], have demonstrated improvement after EFT treatment. The results of the present study are consistent with research showing that treating emotional memories with EFT produces physiological improvements.

\section{CONCLUSIONS}

Clinical reports and accounts from coaches and athletes note that EFT is practiced in professional and college level golf, baseball, and American football. This study indicates that a very brief application of EFT, administered in a compressed time frame similar to the duration of a game, can increase performance in high-performing athletes. The free throw performance of both women and men benefited from EFT. The use of EFT for stress reduction in sports warrants further empirical evaluation. Further study is required to determine if longer courses of EFT produce changes in vertical jump height, or greater improvement in free throw scores. The study of this technique in healthy populations and peak performers opens up new potential applications for Energy Psychology.

\section{ACKNOWLEDGMENTS}

Thanks to psychiatrist and clinical psychologist colleagues who provided advice on study design and on drafts of this paper: Daniel Benor, MD, David Feinstein, PhD, John Freedom, CEHP, Phillip Friedman PhD, Richard Leskowitz, MD, Gregory Nicosia, PhD, Jack Rowe, PhD, Norman Shealy, MD, PhD, and members of the ACEP Research Committee. Special thanks to Greg Warburton, LPC, who arranged access to the OSU teams, assisted in the study, and together with Stacy Vornbrock, LPC, provided treatment. Thanks to Audrey Brooks PhD for data analysis, and to Leane Roffey Line PhD for editorial assistance. Thanks to women's basketball head coach LaVonda Wagner, men's basketball head coach Jay John, and OSU Director of Athletics, Bob DeCarolis, for their cooperation in conducting this study.

\section{REFERENCES}

[1] Feinstein D. Energy psychology: a review of the preliminary evidence. Psychother Theory Res Prac Train 2008; 45(2): 199 213.

[2] Achenbach J. Golfers "tap" into psychology. Golfweek 2007; 25: 60.

[3] Borzi P. Beavers 11, Tar Heels 4; Oregon State one win from a second title. New York Times 2007, June 24; Available from: http://query.nytimes.com

[4] Crist R. Elite athletes and amateurs alike are continually searching for things that can help them attain levels of peak performance. Scottsdale Republic 2005. 
[5] Callahan R. Tapping the healer within: using thought field therapy to Instantly conquer your fears, anxieties, and emotional distress. New York: McGraw-Hill 2000.

[6] Feinstein D, Eden D, Craig G. The promise of energy psychology. New York: Tarcher 2005; p. 108.

[7] Craig G. The EFT Manual. Santa Rosa, CA: Energy Psychology Press 2008.

[8] Rowe J. EFT and golf. Bangor, ME: Booklocker 2009.

[9] EFT Power Training. Available from: http://www.eftpowertraining. com, [Accessed: 5 Oct 2009].

[10] Rowe J. The effects of EFT on long-term psychological symptoms. Counsel Clin Psychol J 2005; 2(3): 104-11.

[11] Lambrou PT, Pratt G. Physiological and psychological effects of a mind/body therapy on claustrophobia. Subtle Energies Energy Med 2003; 14(3): 239 .

[12] Wells S, Polglase K, Andrews HB, Carrington P, Baker AH. Evaluation of a meridian-based intervention, emotional freedom techniques (EFT), for reducing specific phobias of small animals. J Clin Psychol 2003; 59(9): 943-6.

[13] Church D, Geronilla L, Dinter I. The effect of six sessions of EFT on PTSD in veterans: an observational study. Int J Heal Caring 2009; 9: 1 .

[14] Mollon P. Psychoanalytic energy psychotherapy. London: Karnac 2008.

[15] Schulz K. Integrating energy psychology into treatment for adult survivors of childhood Sexual Abuse. Energy Psychol Theory Res Treat 1(1): 13-16.

[16] Brattberg G. Self-administered EFT (Emotional Freedom Techniques) in individuals with fibromyalgia: a randomized trial. Integrative Med 2008; 7(4): 30-35.

[17] Gallo FP. Energy psychology. Boca Raton, FL: CRC Press 1999; p. xi.

[18] Oschman J. Trauma energetics. J Bodyw Mov Ther 2006; 10: 2134.

[19] Church D. The effect of psychotherapy on stress biochemistry: a comparison of EFT and CBT. Paper presented at the annual meeting of the Association for Comprehensive Energy Psychology. Albuquerque, NM 2008

[20] Lane J. The neurochemistry of counterconditioning: acupressure desensitization in psychotherapy. Energy Psychol Theory Res Treat 1(1): 43-59.

[21] Ruden RA. Neurobiological basis for the observed peripheral sensory modulation of emotional responses. Traumatology 2005; 11(3): 145-158.

[22] Church D. The genie in your genes. $2^{\text {nd }}$ ed. Santa Rosa, CA: Energy Psychology Press 2009; p. 227.

[23] Trninić S, Dizdar DE, Luksic E. Differences between winning and defeated top quality basketball teams in final tournaments of European club championship. Coll Antropol 2002; 26(2): 521-31.

[24] Behncke L. Mental skills training for sports: a brief review. Athletic Insight 2004; 6(1): 1-19.
[25] Hamilton SA, Fremouw WJ. Cognitive-behavioral training for college basketball free-throw performance. Cogn Ther Res 1985; 9(4): 479-83

[26] Kearns DW, Crossman J. Effects of a cognitive intervention package on the free-throw performance of varsity basketball players during practice and competition. Percept Mot Skills 1992; 75(3 Pt 2): 1243-53.

[27] Filaire E, Alix D, Ferrand C, Verger M. Psychophysiological stress in tennis players during the first single match of a tournament. Psychoneuroendocrinology 2009; 34(1): 150-7.

[28] Bachman R. In Corvallis, suddenly there came a tapping. The Oregonian 2007.

[29] Whitehead R, Butz JW, Kozar B, Vaughn RE. Stress and performance: an application of Gray's three-factor arousal theory to basketball free-throw shooting. J Sports Sci 1996; 14(5): 393-401.

[30] Moore J, Brylinksky JA. Spectator effect on team performance in college basketball. J Sport Behav 1993; 16: 77-84.

[31] Kuipers H. Training and overtraining: an introduction. Med Sci Sports Exerc 1998; 30(7): 1137-1139.

[32] Williams JH, Klug GA. Calcium exchange hypothesis of skeletal muscle fatigue: a brief review. Muscle Nerve 1995; 18(4): 421-34.

[33] Dougherty KA, Baker LB, Kenney WL. Two percent dehydration impairs and six percent carbohydrate drink improves boys' basketball skills. Med Sci Sports Exerc 2006; 38(9): 1650-8.

[34] Hoffman JR, Stavsky H, Falk B. The effect of water restriction on anaerobic power and vertical jumping height in basketball players. Int J Sports Med 1995; 16(4): 214-8.

[35] Profile: Rick Pitino, 70. Success 1998, October.

[36] Bigland-Ritchie B, Woods JJ. Changes in muscle contractile properties and neural control during human muscular fatigue. Muscle Nerve 1984; 7(9): 691-9.

[37] Busso H, Candau R, Lacour JR. Fatigue and fitness modelled from the effects of training on performance. Eur J Appl Physiol 1994; 69(1): 50-4.

[38] Wolpe J. The Practice of therapy. $2^{\text {nd }}$ ed. New York: Pergamon Press 1973.

[39] Cherkin DC, Sherman KJ, Avins AL, et al. A randomized trial comparing acupuncture, simulated acupuncture, and usual care for chronic low back pain. Arch Intern Med 2009; 169(9): 858-66.

[40] Feinstein D. Energy psychology in disaster relief. Traumatology 2008; 14(1): 27-39.

[41] Theodorakis Y. Self-talk in a basketball shooting task. Percept Mot Skills 2001; 92(1): 309-15.

[42] Raglin J. Anxiety and sports performance. Exerc Sport Sci Rev 1992; 20(1): 243.

[43] Robazza C, Bortoli L. Intensity, idiosyncratic content and functional impact of performance-related emotions in athletes. J Sports Sci 2003; 21(3): 171-89.

[44] Church D. Evaluating physiological markers of emotional trauma: a randomized controlled blind trial of mind-body therapies. Paper presented at the annual meeting of the Association for Comprehensive Energy Psychology; Albuquerque, NM 2008.

(C) Church et al.; Licensee Bentham Open.

This is an open access article licensed under the terms of the Creative Commons Attribution Non-Commercial License (http://creativecommons.org/licenses/by-nc/3.0/) which permits unrestricted, non-commercial use, distribution and reproduction in any medium, provided the work is properly cited. 